\title{
Solvatochromic behavior of the electronic absorption spectra of some azo derivatives of amino pyridines
}

\author{
Mamdouh S. Masoud ${ }^{\mathrm{a}, *}$, Alaa E. Ali ${ }^{\mathrm{b}}$, \\ Medhat A. Shaker ${ }^{\mathrm{b}}$, Mohamed Abdul Ghani ${ }^{\mathrm{c}}$ \\ ${ }^{a}$ Chemistry Department, Faculty of Science, Alexandria University, Alexandria, Egypt \\ ${ }^{\mathrm{b}}$ Physics and Chemistry Department, Faculty of Education, Damanhour, Alexandria University, Egypt \\ ${ }^{c}$ Chemistry Department, Faculty of Science, Beirut Arab University, Beirut, Lebanon
}

Received 20 October 2003; accepted 18 February 2004

\begin{abstract}
The electronic absorption spectra of a series of the entitled eight compounds containing groups with variable electronic characters were recorded. The solvents were selected to cover a wide range of parameters (refractive index, dielectric constant and hydrogen bonding capacity). The electronic transitions are assigned and the solvent induced spectral shifts have been analyzed in relation to the different solute-solvent interaction mechanisms using computational chemistry. The regression analysis is applied for correlation parameters. The phenomenon of tautomerism is explained. The electronic character of the substituent as well as the chemistry of the solvent are the major factors for the solvatochromic behavior.
\end{abstract}

(C) 2004 Elsevier B.V. All rights reserved.

Keywords: Solvatochromic behavior; Amino pyridines; Azo compounds

\section{Introduction}

Some azo compounds have recently attracted increasing attention due to their many emerging applications. Azo molecules are characterized by a high photo induced anisotropy [1], being excellent photo aligning substrates for liquid crystals [2] and highly efficient photorefractive media [3]. The photochemistry [4] and microstructuring $[5,6]$ of several classes of azo compounds have been investigated in earlier studies. Their photosensitivity and the superior structuring properties are mainly due to the lability of substituents binding to the $\mathrm{N}=\mathrm{N}$ groups. The effect of solvent on the UV-Vis spectra of azo compounds has been extensively studied [7-11]. Azo dyes are normally known to show a positive solvatochromism [12-19]. The first examples of negative solvatochromism in neutral azo dyes containing both strongly electron-donating and -withdrawing moieties were reported [20]. In this work, we employ substituted derivatives of the parent azo compound having both strong electron-donating and -withdrawing moieties in a molecule

\footnotetext{
* Corresponding author.

E-mail address: drmsmasoud@yahoo.com (M.S. Masoud).
}

as probes to examine the effect of molecular structure on their UV-Vis spectra. The investigated azo compounds are derived from 2-amino-3-hydroxy pyridine. Moreover, it was of interest to examine also the solvatochromic behavior of these compounds. The analysis of the current data has shown that both positive and negative solvatochromism correlate to the identity of the substituent and the solvent parameters as well. Herein, the solvatochromism of these azo dyes is tested using various solvents. The solvents are selected to have a wide variety of solvent parameters. The solvent induced spectral shifts of the studied compounds have been analyzed and correlated to the solvent parameters using statistical techniques.

\section{Experimental}

The azo dyes derived from 2-amino-3-hydroxy pyridine were synthesized by the usual diazotisation-coupling reaction [21]. The UV-Vis spectra were measured with a Shimadzu UV 160A spectrometer. The solvents used were of spectrophotometeric grade. They were purchased from Sigma-Aldrich and were used as received. 


\subsection{Regression analysis calculations}

Different one-, two- and three-parameter equations are applied here using suitable combinations between the solvent polarity parameters $E, K, M, J, H$ and $N$ as reported before [22]. The parameter $E$ is sensitive to both solvent-solute hydrogen bonding and dipolar interactions [23]. The dielectric function, $K$, of Kirkwood adequately represents the dipolar interactions [24]. The functions $H$ and $J$ have been introduced to account for the non-specific solute-solvent interactions such as dispersion and dipolar effects [25]. The functions $M$ and $N$ account for the solute permanent dipole-solvent induced dipole and solute permanent dipole-solvent permanent dipole interactions, respectively [26]. In the present work, the observed peak position of an absorption band $Y$ in a given solvent has been expressed as a linear function of different solvent polarity parameters $X_{n}$, as follows:

$Y=a+b_{1} X_{1}+b_{2} X_{2}+\ldots+b_{n} X_{n}$

Eq. (1) is amenable to solution for the intercept $a$ and the coefficients $b_{1}, b_{2}, \ldots, b_{n}$ by multiple regression technique. The regression intercept $a$ has been considered as the peak position in the gas phase spectra [27]. A program of statistical package of social sciences (SPSS) has been used on a Pentium IV computer at the Faculty of Education in Damanhour, Egypt. The residual error $S$ in a least squares that fits the multi-linear Eq. (1) is:

$S=\sum_{i=1}^{m}\left(Y_{i}-a-b_{1} X_{1 i}-b_{2} X_{2 i}-\ldots-b_{n} X_{n i}\right)^{2}$

The least-squares criterion requires that $S$ be minimized. This results in sets of equations that can be solved by use of standard matrix methods.

\section{Results and discussion}

Azo dyes contain intramolecular charge-transfer chromophores and, therefore, their UV-Vis absorption bands depend on the combination of electron-donating and -withdrawing moieties in the molecules. The azo dyes studied have both strong electron-donating and -withdrawing moieties. These dyes are derived from 2-amino-3-hydroxy pyridine. They are 2-amino-6-phenylazo-pyridin-3-ol (I), 2-amino-6-p-tolylazo-pyridin-3-ol (II), 2-amino-6-(4-fluorophenylazo)-pyridin-3-ol (III), 4-(2-amino-3-hydroxy-pyridin-6-ylazo)-benzoic acid (IV), 2-amino-6-(4-bromo-phenylazo)-pyridin-3-ol (V), $N$-[4-(2-amino-3-hydroxy-pyridin-6ylazo)-phenyl]-acetamide (VI), 4-(2-amino-3-hydroxy-pyridin-6-ylazo)-benzoic acid ethyl ester (VII) (Fig. 1). The solvatochromic behavior of these azo dyes is examined using various solvents. The solvents are selected to have a wide variety of solvent parameters such as dielectric constant, $d$, refractive index, $n$, and hydrogen bonding capacity to

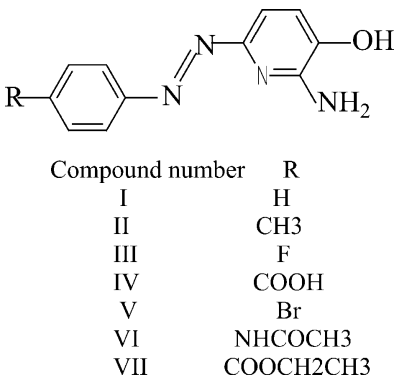

Fig. 1. Structures and symbols of the investigated compounds.

Table 1

Solvent parameters used in the spectral correlation equations

\begin{tabular}{lllllll}
\hline Solvent & $n$ & $d$ & $E$ & $K$ & $M$ & $N$ \\
\hline$n$-Hexane & 1.375 & 1.9 & 30.9 & 0.188 & 0.188 & 0.00191 \\
Toluene & 1.497 & 2.4 & 33.9 & 0.241 & 0.226 & 0.026 \\
Diethyl ether & 1.353 & 4.2 & 34.6 & 0.340 & 0.178 & 0.300 \\
Ethyl acetate & 1.372 & 6.0 & 38.1 & 0.385 & 0.185 & 0.398 \\
Acetone & 1.359 & 20.7 & 42.2 & 0.465 & 0.180 & 0.648 \\
DMSO & 1.478 & 48.9 & 45.0 & 0.485 & 0.221 & 0.658 \\
2-Propanol & 1.377 & 18.3 & 48.6 & 0.460 & 0.187 & 0.622 \\
Ethanol & 1.361 & 24.3 & 51.9 & 0.470 & 0.181 & 0.665 \\
Methanol & 1.329 & 32.6 & 55.5 & 0.477 & 0.169 & 0.710 \\
Water & 1.333 & 78.5 & 63.1 & 0.491 & 0.171 & 0.757 \\
\hline
\end{tabular}

make comparison easier and permit a good understanding of solvatochromic behavior. Table 1 shows the values of these solvent parameters [28].

The electronic absorption spectra of the investigated azo compounds are collected in (Table 2). The UV-Vis absorption spectrum of azo dye (I), the parent compound, in $n$-hexane exhibits two absorption bands with $\lambda_{\max }$ at 241 and $481 \mathrm{~nm}$ (Fig. 2). The absorption band corresponds to the lowest transition at $481 \mathrm{~nm}$ and is assigned to the partly forbidden $\left(\mathrm{n} \rightarrow \pi^{*}\right)$ transition. This band shows positive solvatochromism (bathochromic shift) upon increasing the solvent polarity. This means that a pronounced change in position of an electronic absorption band is accompanying a change in the polarity of the medium. This observed behavior is accounted as that molecules in the ground state and in the excitation state indicate different polarities. This

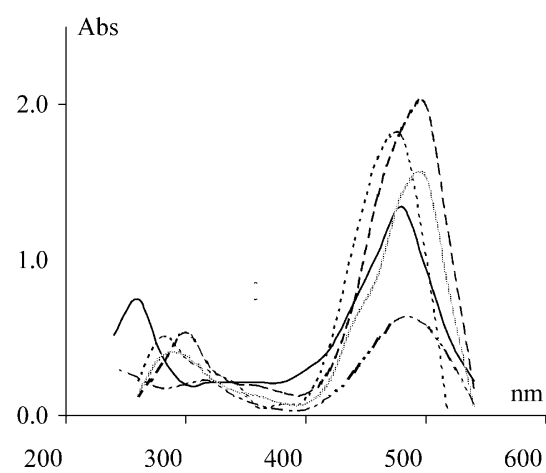

Fig. 2. The electronic absorption spectra of azo dye (I) in different solvents. 
Table 2

The observed $\lambda_{\max }$ values of the investigated azo compounds

\begin{tabular}{|c|c|c|c|c|c|c|c|c|c|c|c|}
\hline $\mathrm{R}$ & $n-\mathrm{C}_{6} \mathrm{H}_{14}$ & $n-\mathrm{C}_{5} \mathrm{H}_{12}$ & $\mathrm{Et}_{2} \mathrm{O}$ & $\left(\mathrm{CH}_{3}\right)_{2} \mathrm{CO}$ & EtOAc & $\mathrm{C}_{6} \mathrm{H}_{5} \mathrm{CH}_{3}$ & $\left(\mathrm{CH}_{3}\right)_{2} \mathrm{COH}$ & EtOH & $\mathrm{MeOH}$ & DMSO & $\mathrm{H}_{2} \mathrm{O}$ \\
\hline \multirow[t]{7}{*}{$\mathrm{H}$} & & & & & & & & 206 & 204 & & \\
\hline & 241 & 227 & 232 & & 246 & & 229 & 233 & 232 & & \\
\hline & & 279 & 273 & & 267 & & & & & & \\
\hline & & & 294 & & 282 & 288 & 286 & 297 & 287 & 292 & \\
\hline & & & & & 308 & & & & & & \\
\hline & & & & & 339 & & & & & 359 & 321 \\
\hline & 481 & 473 & 474 & 472 & 477 & 487 & 491 & 489 & 490 & 492 & 487 \\
\hline \multirow[t]{5}{*}{$\mathrm{CH}_{3}$} & 235 & 227 & 224 & & & & 226 & 208 & 206 & 260 & \\
\hline & & 255 & 252 & & 251 & & 255 & & & & \\
\hline & & 261 & 294 & & 282 & 285 & 278 & & & & \\
\hline & & 279 & 307 & & & & & & & & \\
\hline & 485 & 470 & 485 & 492 & & 493 & 497 & 495 & 497 & 500 & 490 \\
\hline \multirow[t]{5}{*}{$\mathrm{F}$} & & & & & & & 218 & 208 & 207 & & \\
\hline & 237 & 227 & 245 & & 251 & & 249 & 255 & & & \\
\hline & & 285 & 273 & & 282 & 284 & 278 & 291 & 279 & 282 & \\
\hline & & & 300 & 477 & & & & & & & \\
\hline & 464 & 467 & 476 & & 472 & 482 & 488 & 483 & 484 & 490 & 487 \\
\hline \multirow[t]{4}{*}{$\mathrm{COOH}$} & 237 & - & & & & & 222 & - & & & \\
\hline & & & 273 & & 277 & & 271 & & & & \\
\hline & & & 297 & & 397 & & & & & & \\
\hline & 533 & & 476 & 477 & 477 & 487 & 488 & & 487 & 492 & 518 \\
\hline \multirow[t]{6}{*}{$\mathrm{Br}$} & & & & & & & 226 & 206 & 200 & & - \\
\hline & & 230 & 230 & & 249 & & 238 & 239 & 235 & & \\
\hline & 338 & 300 & 294 & & 297 & 284 & 258 & 264 & 258 & & \\
\hline & & & & & & & 296 & 300 & 297 & & \\
\hline & 364 & 354 & 355 & 359 & 359 & 363 & 341 & 365 & 355 & & \\
\hline & 477 & 473 & 479 & 477 & 477 & 485 & 491 & 486 & 485 & 495 & \\
\hline \multirow[t]{6}{*}{$\mathrm{NHCOCH}_{3}$} & & - & & & & & & 212 & 205 & & \\
\hline & 252 & & 242 & & 249 & & 245 & 254 & 251 & & 246 \\
\hline & & & 270 & & & 282 & 270 & & & 272 & \\
\hline & & & 306 & 292 & 297 & & & & & & \\
\hline & & & & & 415 & & & & & & \\
\hline & & & 494 & & 495 & 500 & 499 & 500 & 497 & 518 & \\
\hline \multirow[t]{4}{*}{$\mathrm{COOCH}_{2} \mathrm{CH}_{3}$} & & & 233 & & 231 & & & 209 & 205 & & \\
\hline & 276 & 276 & 279 & & 277 & 285 & 282 & 285 & 287 & & 282 \\
\hline & & & 306 & & & 316 & 301 & 297 & & 292 & \\
\hline & & 467 & 476 & 456 & 472 & 487 & 486 & 491 & 492 & 497 & \\
\hline
\end{tabular}

interpretation involves the highly simplifying assumptions that dyes with non-polarized ground state are more strongly polarized in protic solvents, because the high-energy, polar structure of the excitation state is stabilized. The excited state is lowered. The ground state is hardly affected. The energy difference between ground and excited states is decreased and the excitation energy is decreased. The approximation of the energy levels expresses itself in a bathochromic shift of the spectrum with increasing polarity of the solvent. A negative solvatochromism (hypsochromic shift) is noticed in solvents like $n$-pentane, diethyl ether, acetone and ethyl acetate. Those non-protic solvents destabilize the polarized electronic state. This leads to a hypsochromic shifting of the spectrum with decreasing solvents polarity. The other absorption band corresponding to the highest energy centered at $241 \mathrm{~nm}$ is localized in the aromatic rings and is due to a $\left(\pi \rightarrow \pi^{*}\right)$ transition. This band shows a negative solva- tochromism in other solvents except diethyl ether. A third band appears in the spectrum of the parent azo dye (I) at $279 \mathrm{~nm}$ in $n$-pentane and shows a positive solvatochromism in all other solvents and disappears in both $n$-hexane and water. Azo compounds with an electron withdrawing group on one side of the molecule and an electron donating group on the other side are an example of the so-called push-pull molecules which are of interest in the field of non-linear optical materials $[20,29]$. A solvatochromism occurred in the lowest energy $\left(\mathrm{n} \rightarrow \pi^{*}\right.$ ) absorption upon increasing the push-pull character of the substituents on the aryl azo component. A solution in $n$-hexane solvent of azo dye (II), where the substituent is the strong electron donating methyl group can be characterized by an absorption spectrum with an intense transition centered at $485 \mathrm{~nm}$ and weak broad band at $235 \mathrm{~nm}$. A positive solvatochromism is observed for the $\left(\mathrm{n} \rightarrow \pi^{*}\right)$ absorption in case of methyl substitution, 


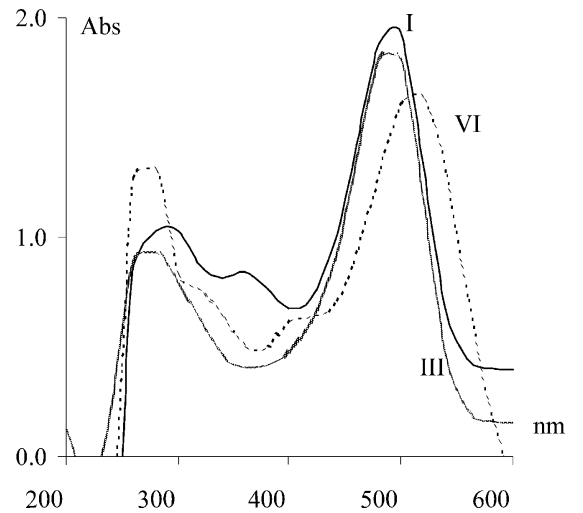

Fig. 3. Substituent effects on the peak positions of compounds I, III and VI.

whereas a negative solvatochromism is observed for this band in case of fluorine, azo dye (III), and bromine substitution, azo dye (V). The hypsochromic shift in this band follows the order $\mathrm{F} \gg \mathrm{Br}$ in an agreement with the order of their electronegativities. The same trend in the solvatochromic behavior of $\left(\mathrm{n} \rightarrow \pi^{*}\right)$ absorption for these azo dyes is observed in solvents like isopropanol, methanol, ethanol and toluene. Absorption spectra for the azo dyes with various electron donating or accepting abilities are shown in (Fig. 3). The UV-Vis spectrum of the azo dye (V) in $n$-pentane exhibits two absorption bands. A band is found at about $354 \mathrm{~nm}$ assigned to a $\left(\pi \rightarrow \pi^{*}\right)$ transition which is red shifted in all other solvents. While blue shifts are observed in the second absorption band which is found at wavelengths shorter than $300 \mathrm{~nm}$ and assigned to a $(\pi \rightarrow$ $\left.\sigma^{*}\right)$ transition. The band with $\lambda_{\max }$ at $533 \mathrm{~nm}$ in $n$-hexane in the spectrum of azo dye (IV), where the substituent in this case is the carboxyl group, is assigned for the (n $\left.\rightarrow \pi^{*}\right)$ electronic transition. A negative solvatochromism is noticed in all other solvents. The spectral shifts decrease with gradual introduction of the more polar solvent. In these solvents the amino and hydroxyl groups in the pyridine moiety will be the proton acceptor center in the process of hydrogen bonds formation whereas the solvents that have the ability to form hydrogen bonds are the proton donors. Now going to solvents which can be considered as dipolar aprotic media such as ethyl acetate, diethyl ether, and dimethyl sulfoxide. These solvents can act as proton acceptor in hydrogen bond formation with the acidic carboxyl group of the azo dye (IV) as proton donor in this case. Also, these solvents have relatively high dipole moments. Dimethyl sulfoxide differs from the other group of solvents used in that its dielectric constant and its polarizability as measured by the refractive index are appreciably greater than those of the other solvents. The dipole-dipole interactions with the solute molecules especially in their excited states will encounter for the blue shifts observed in these solvents. The energy of a charge transfer will increase as the strength of such hydrogen bonds increases. The observed marginal shift in the absorption maximum for the azo dyes may arise from contributions other than solvatochromic effects. However, in addition to these shifts, a significant band broadening for the azo compounds with increasing solvent polarity was observed. It is concluded that solvatochromism is a useful indicator of the strength of hydrogen bonding and has even been a good tool to assess their strengths. Several one-, twoand three-parameter equations have been used to correlate the spectral shifts with various empirical solvent polarity parameters, using the multiple linear regression technique. Each of the solvent parameter used has a fixed relative sensitivity to each of the various interaction mechanisms. The multiple correlation coefficient (MCC), has been used in a one-tail test to obtain the level of significance for each test. Also, the small value (near zero) of the significance parameter $(P)$ means the correlation is good. Table 3 is a model representative example showing the results of regression analysis for the $\left(\pi \rightarrow \pi^{*}\right)$ transition of azo (III). Analysis of solvent spectral shifts using one-parameter equations indicated that for the $\left(\pi \rightarrow \pi^{*}\right)$ transition of azo (III), the solvent polarity parameters $K$ and $N$, which are functions

Table 3

The results of regression analysis for the $\left(\pi \rightarrow \pi^{*}\right)$ transition of azo (III)

\begin{tabular}{|c|c|c|c|c|c|c|c|}
\hline & $a_{0}$ & $a_{1}$ & $a_{2}$ & $a_{3}$ & $a_{4}$ & $P$ & MCC \\
\hline$K$ & 226.816 & 55.843 & & & & 0.020 & 0.872 \\
\hline$M$ & 355.395 & -587.571 & & & & 0.551 & 0.131 \\
\hline$N$ & 238.096 & 23.414 & & & & 0.028 & 0.843 \\
\hline$E$ & 221.876 & 0.625 & & & & 0.084 & 0.685 \\
\hline$K, M$ & 256.070 & 54.163 & & & & 0.120 & 0.880 \\
\hline$K, N$ & 219.621 & 92.190 & -15.608 & & & 0.123 & 0.877 \\
\hline$K, E$ & 228.053 & $-8.62 \mathrm{E}-2$ & 62.037 & & & 0.126 & 0.874 \\
\hline$M, N$ & 280.630 & -229.475 & 22.517 & & & 0.138 & 0.862 \\
\hline$M, E$ & 307.230 & -459.717 & 0.604 & & & 0.236 & 0.764 \\
\hline$N, E$ & 248.302 & 35.081 & -0.364 & & & 0.135 & 0.865 \\
\hline$K, M, N$ & 246.211 & 77.259 & -127.310 & -9.786 & & 0.428 & 0.882 \\
\hline$K, M, E$ & 254.818 & $-2.09 \mathrm{E}-2$ & 55.757 & -147.523 & & 0.431 & 0.880 \\
\hline$M, N, E$ & 272.399 & -144.824 & 31.375 & -0.266 & & 0.447 & 0.872 \\
\hline$K, N, E$ & -68.826 & -510.560 & 4.202 & 942.952 & & 0.320 & 0.935 \\
\hline$K, M, N, E$ & -163.360 & 1504.436 & -566.754 & -849.969 & 7.304 & 0.000 & 1.000 \\
\hline
\end{tabular}


of dipolar interactions and solute permanent dipole-solvent permanent dipole interactions, respectively, have shown a good correlation with the solvent spectral shifts (Table 3). These solvent parameters have the highest calculated MCC values among all other parameters. The calculated MCC values are 0.872 and 0.843 which are indicative that the solvent refractive index and dielectric constant are playing the major role in determining the solvent spectral shifts. For $\left(\mathrm{n} \rightarrow \pi^{*}\right)$ transition in azo (III), the solvent polarity parameter $E$, which is related to the solvent ability to form hydrogen bond with the solute molecules is the only parameter that had a good correlation with the solvent spectral shifts with a calculated MCC value of 0.758 . In the $(\pi \rightarrow$ $\left.\pi^{*}\right)$ transition of azo (II) the parameter $M$, which is related to the solvent refractive index has the highest calculated MCC value among all other parameters. Its calculated MCC value is 0.611 . The solvatochromic shifts for all other peaks in the investigated compounds have poor correlations on analyzing the solvent spectral shifts using one-parameter equations. The correlation of the two-parameter equations with the solvent spectral shifts was also studied and gave, as expected, better fit to these spectral shifts than the corresponding one-parameter fits. For the compound (III), the two-parameter combinations $K M$ and $K N$ correlate the spectral shifts for the $\left(\pi \rightarrow \pi^{*}\right)$ transition with calculated MCC values of 0.880 and 0.877 , respectively (Table 3 ) while the two-parameter combinations $M E$ and $N E$ showed the best correlation among all other different two-parameter for the $\left(\mathrm{n} \rightarrow \pi^{*}\right)$ transition with calculated MCC values of 0.898 and 0.785 , respectively. Thus, the determinations of the solvent spectral shifts are controlled by mainly the solvent ability to form hydrogen bonds with the solute molecules as well as both the solvent dielectric constant and the solvent refractive index. For compound (VI), the two-parameter combinations $K M$ and $K N$ correlate the spectral shifts for the $\left(\mathrm{n} \rightarrow \pi^{*}\right)$ transition with calculated MCC values of 0.929 and 0.923 , respectively. The solvent spectral shifts in this transition are controlled by both the solvent dielectric constant and the solvent refractive index. Addition of a third solvent parameter to the two-parameter equations always gave rise to improvements in the correlation with the solvent induced spectral shifts. In most cases, the different three-parameter combinations have been selected on the basis of the results of the two-parameter combinations discussed before. In a test for the significance of a one-tail test, the level of significance for all these different three-parameter combinations were found to be above $90 \%$. This indicated that specific solute-solvent interactions in particular hydrogen bonding and non-specific solute-solvent interactions such as dispersion and dipolar effects had provided a reasonable model for describing the solvent induced spectral shifts in a predictive manner.

\section{References}

[1] O. Yaroshchuk, T. Sergan, J. Lindau, S.N. Lee, L.C. Chien, J. Chem. Phys. 114 (2001) 5330.

[2] W. Gibbons, P. Shannon, S. Shao-Tang, B. Swetlin, Nature 351 (1991) 49.

[3] S. Bartkiewicz, K. Matczyszyn, A. Miniewicz, F. Kajzar, Opt. Commun. 187 (2001) 257.

[4] A. Stasko, V. Adamcik, T. Lippert, A. Wokaun, J. Dauth, O. Nuyken, Makromol. Chem. 194 (1993) 3385.

[5] T. Lippert, J. Stebani, J. Ihlemann, O. Nuyken, A. Wokaun, J. Phys. Chem. 97 (1993) 12296.

[6] T. Lippert, A. Wokaun, J. Stebani, O. Nuyken, J. Ihlemann, J. Angew. Makromol. Chem. 213 (1993) 127.

[7] M.S. Masoud, A.A. Abdallah, F.M.N. Kheiri, Indian Textile J. 94 (1983) 103.

[8] M.S. Masoud, E.A. Khalil, Polym. J. Chem. 65 (1991) 933.

[9] M.S. Masoud, S.S. Hagag, H.M. El-Nahas, N. Abd El-Hi, Acta Chim. Hungarica 130 (1993) 783.

[10] M.S. Masoud, A.A. Hasanein, A.K. Ghonaim, E.A. Khalil, A.A. Mahmoud, Zeitschrift Phy. Chem. Bd. 209 (1999) 223.

[11] M.S. Masoud, H.H. Hammad, Spectrochim. Acta (Part A) 57 (2001) 977.

[12] M.G. Hutchings, P. Gregory, J.S. Campbell, A. Strong, J.-P. Zamy, A. Lepre, A. Mills, Chem. Eur. J. 3 (1997) 1719.

[13] C. Machado, M.G. Nascimento, M.C. Rezende, J. Chem. Soc., Perkin Trans. 2 (1994) 2539.

[14] A.T. Peters, A. Gbadamosi, J. Chem. Technol. Biotechnol. 53 (1992) 301.

[15] D.M. Shin, K.S. Schanze, D.G. Whitten, J. Am. Chem. Soc. 111 (1989) 8494.

[16] D.M. Shin, D.G. Whitten, J. Am. Chem. Soc. 110 (1988) 5206.

[17] S. Kobayashi, H. Kamei, H. Yokoyama, Chem. Phys. Lett. 138 (1987) 333.

[18] N. Nishimura, T. Tanaka, M. Asano, Y. Sueshi, J. Chem. Soc., Perkin Trans. 2 (1986) 1839.

[19] H. Epperlein, J. Mustroph, J. Prakt. Chem. 322 (1980) 305.

[20] J.J. Kim, K. Funabiki, H. Muramatsu, K. Shibata, S.H. Kim, H. Shiozaki, H. Hartmannd, M. Matsui, Chem. Commun. 9 (2000) 753.

[21] A.I. Vogel, A Text Book of Practical Organic Chemistry, third ed., Longmans, London, 1961.

[22] M.A. Shaker, M.A. Khalifa, Alex. J. Pharm. Sci. 9 (1995) 159.

[23] C. Reichardt, Angew. Chem. 4 (1965) 29.

[24] J.G. Kirkwood, J. Chem. Phys. 2 (1934) 351.

[25] J.G. David, H.E. Hallam, Spectrochim. Acta 23A (1967) 593.

[26] E.G. McRae, J. Phys. Chem. 61 (1957) 562.

[27] L.J. Hilliard, D.S. Foulk, H.S. Gold, Anal. Chim. Acta 133 (1981) 319.

[28] F.W. Fowler, A.R. Katritzky, R.J.D. Rutherford, J. Chem. Soc. (B) (1971) 460.

[29] J.J. Kim, K. Funabiki, H. Muramatsu, K. Shibata, S.H. Kim, H. Shiozaki, H. Hartmann, M. Matsui, J. Chem. Soc., Perkin Trans. 2 (2001) 379. 